\title{
Synthesis and In Vitro Evaluation of Chitosan- Thioglycolic Acid Conjugates
}

\author{
Andreas Bernkop-Schnürch* and Thorid E. Hopf \\ Institute of Pharmaceutical Technology and Biopharmaceutics, Center of Pharmacy, University of \\ Vienna, Althanstr. 14, A-1090 Vienna, AUSTRIA / Europe
}

Summary: The cationic thiomer chitosan-thioglycolic acid (TGA) shows excellent mucoadhesive features. In order to deepen the knowledge concerning this new excipient the optimization of its synthesis and a detailed characterization of its properties was the objective of this study Mediated by increasing quantities of a carbodiimide, thioglycolic acid was covalently attached to chitosan forming amide bonds with the primary amino groups of the polymer Determined with Ellman's reagent, $38 \pm 3,104 \pm 2,685 \pm 43$, and $885 \pm 7 \mu \mathrm{mol}$ thiol groups $(\mathrm{n}=3 ; \pm \mathrm{SD}$ ) were bound per gram polymer at carbodiimide concentrations of $50,75,100$, and $125 \mathrm{mM}$, respectively. The immobilized thiol groups displayed a comparatively higher reactivity to form disulfide bonds than the thiol groups in a corresponding mixture of chitosan and free unconjugated TGA. In an aqueous $0.5 \%$ $(\mathrm{m} / \mathrm{v})$ chitosan-TGA gel $59 \pm 5 \%$ of the thiol groups formed disulfide bonds within 6 hours at $\mathrm{pH}$ 6.0 , whereas merely $5 \pm 3 \%$ were oxidized in the corresponding physical mixture of chitosan and TGA. Diffusion studies showed that the modified polymer was capable of binding cysteine and cysteine methyl ester. The result supports the theory that the improved mucoadhesive properties of thiolated chitosan are based on the formation of disulfide bonds with cysteine moieties of mucus glycoproteins. Because of its availability via an efficient synthetic pathway and its mucoadhesive properties based on the capability to bind cysteine subunits, chitosan-TGA seems to be a promising new excipient for various drug delivery systems

Keywords: chitosan, thioglycolic acid, thiomer, cysteine binding, disulfide bonds

\section{Introduction}

Within the last years a promising new type of mucoadhesive hydrophilic polymers has been introduced into the pharmaceutical literature. Because of the immobilization of thiol moieties on well established hydrophilic polymers such as poly(acrylates) and cellulose derivatives their 
mucoadhesive properties are strongly improved ${ }^{\text {e.g. 1-3 }}$ Thiolated polymers - or so called thiomersare believed to form covalent bonds with cysteine-rich subdomains of mucus glycoproteins, ${ }^{4}$ whereas the binding of so far used mucoadhesive polymers has merely been based on non-covalent bonds such as hydrogen bonds and ionic interactions. Moreover, thiolated polymers show improved cohesive properties, as a simple oxidation process in aqueous media leads to the formation of interand/or intra-chain disulfide bonds within the polymeric network. ${ }^{5}$ Recently, an enzyme inhibitory capability ${ }^{6}$ as well as a permeation enhancing effect ${ }^{7}$ could be shown for thiomers, which render them useful particularly for the non-invasive application of peptide drugs. A significant reduction in the glucose level of diabetic mice, for instance, was achieved by the oral administration of insulin microtablets based on a thiolated polymer. ${ }^{8}$

Among thiomers a chitosan-thioglycolic acid (TGA) conjugate has received considerable attention, as it displays more than 10 -fold higher mucoadhesive properties on freshly excised intestinal mucosa compared to unmodified chitosan. ${ }^{9}$ In order to improve the knowledge of thiolated chitosan it was the aim of this study to optimize its synthesis and to carry out further in vitro evaluations for its characterization. In particular, the binding capability for cysteine and a derivative thereof will be evaluated within this study, as the covalent binding of thiomers to mucus glycoproteins seems to take place via cysteine moieties within the mucin amino acid sequence. Apart from the formation of disulfide bonds between thiomers and cysteine groups of mucus glycoproteins, the formation of inter- and/or intra-chain disulfide bonds within chitosan-TGA itself seems to be another important capability of this novel excipient. Because of this cross-linking process the cohesive properties of chitosan can be improved rendering it useful e.g. as tablet binder or for the manufacture of particulate delivery systems which are stabilized by disulfide bonds within the polymeric network A detailed evaluation of the influence of the covalent attachment of TGA on the reactivity of thiol groups being responsible for this crosslinking process was therefore also the objective of this study

\section{Results}

\section{Influence of the carbodiimide concentration on the coupling reaction}

Thioglycolic acid (TGA) was attached covalently to the poly-D-glucosamine chitosan. This was achieved by the formation of amide bonds between the primary amino groups of the polymer and the carboxylic acid group of thioglycolic acid leading to substructures as shown in Fig. 1. In contrast to a previous study focusing on the influence of the $\mathrm{pH}$ on the coupling reaction between chitosan and $\mathrm{TGA}^{9}$, the impact of the carbodiimide concentration on the amount of covalently attached TGA was determined. As a $\mathrm{pH}$ of 5.0 proved to be best for synthesis, ${ }^{9}$ all coupling reactions were performed at this $\mathrm{pH}$, with varying concentrations of carbodiimide The results 
demonstrated a clear correlation between the carbodiimide concentration during the coupling reaction and the coupling rate.

Figure 1. Chemical substructure of the chitosan-TGA conjugate

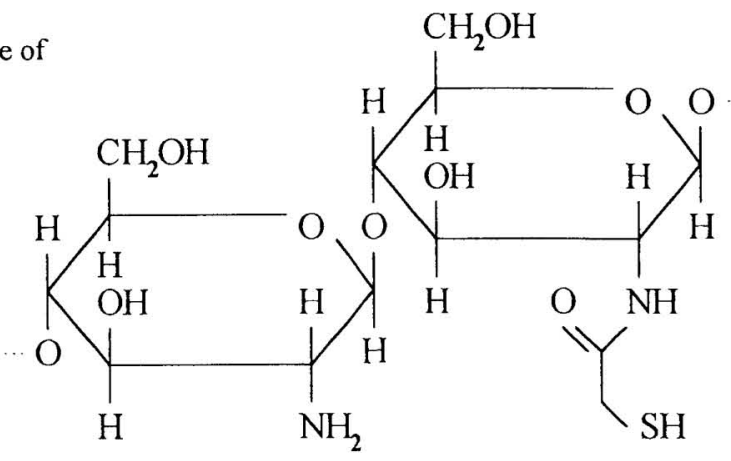

The more carbodiimide was added, the higher was the amount of covalently bound TGA. The results of this study are shown in Fig. 2. During the coupling reaction the carboxylic acid group of TGA was activated by the carbodiimide forming an $O$-acylurea derivative as intermediate product Although the theoretical maximum concentration of primary amino groups on chitosan was $62 \mathrm{mM}$, a higher TGA concentration i.e. $87 \mathrm{mM}$ was used for all reactions. At carbodiimide concentrations of $50 \mathrm{mM}$ and $75 \mathrm{mM}$ consequently not all carboxylic acid groups of TGA could be activated Calculations of the theoretical maximum of TGA which can be immobilized to chitosan as shown in Table 1, however, demonstrated that only a minor amount of added carbodiimide mediated the formation of amide bonds.

Figure 2. Influence of increasing carbodiimide (EDAC) concentrations on the amount of polymer immobilized thiol groups. Indicated bars are means \pm SD of at least three experiments

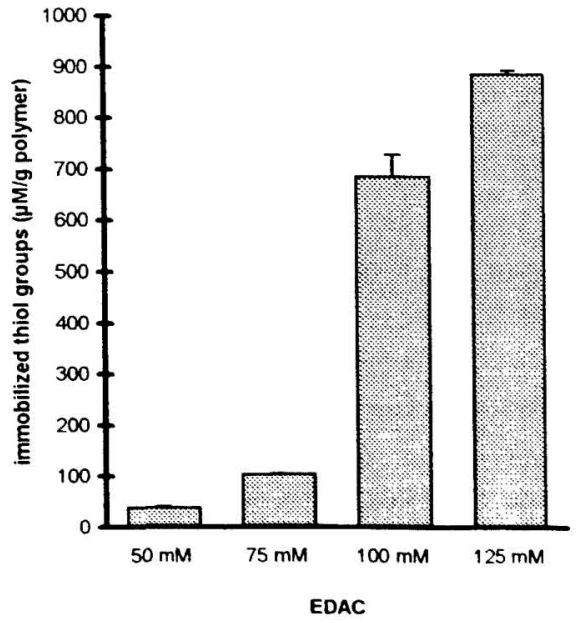


Besides the formation of amide bonds, carbodiimides mediate also the formation of ester bonds. ${ }^{\mathbf{1 0}}$ This was shown e.g. for carboxymethylcellulose-cysteine conjugates leading to a constant raise in the $\mathrm{pH}$ during the coupling reaction, as the concentration of carboxylic acid groups of cysteine decreased by the formation of esters ${ }^{2}$ During the coupling reaction of chitosan-TGA conjugates, however, the $\mathrm{pH}$ remained constant at 5.0, indicating that only a negligible amount of esters are formed between the hydroxyl groups of chitosan and the carboxylic acid group of TGA. The addition of $5 \mathrm{mM}$-hydroxysulfosuccinimide, which is known to enhance carbodiimide mediated coupling reactions ${ }^{\text {"1 }}$ did not lead to higher yields of immobilized thiol groups

Table 1. Influence of the carbodiimide (EDAC) concentration on the yield of thiol moieties, which were covalently attached to chitosan.

\begin{tabular}{ccc}
\hline \hline $\begin{array}{c}\text { EDAC concentration } \\
\text { during the coupling } \\
\text { reaction }\end{array}$ & $\begin{array}{c}\text { Theoretical maximum of thiol } \\
\text { groups on the polymer conjugate }\end{array}$ & $\begin{array}{c}\text { Determined thiol groups on the } \\
\text { polymer conjugate (\% of the } \\
\text { theoretical maximum) }\end{array}$ \\
\hline $50 \mathrm{mM}$ & $5 \mathrm{mMol} / \mathrm{g}$ & $\mathbf{0 . 8 \%}$ \\
$75 \mathrm{mM}$ & $6.2 \mathrm{mMol} / \mathrm{g}$ & $\mathbf{1 . 7 \%}$ \\
$100 \mathrm{mM}$ & $6.2 \mathrm{mMol} / \mathrm{g}$ & $\mathbf{1 1 . 1 \%}$ \\
$125 \mathrm{mM}$ & $6.2 \mathrm{mMol} / \mathrm{g}$ & $\mathbf{1 4 . 3 \%}$ \\
\hline \hline
\end{tabular}

\section{Comparison of the disulfide bond formation kinetic within chitosan-TGA conjugate and a chitosan / TGA mixture}

The formation of disulfide bonds within chitosan-TGA conjugate was compared with that of free unconjugated TGA in combination with chitosan. The results of this study demonstrated a continuous oxidation process within the chitosan-TGA conjugate, whereas free TGA remained almost stable at $\mathrm{pH} 6.0$. The results are illustrated in Fig. 3. The $\mathrm{pK}_{\mathrm{a}}$ for the thiol moiety of thioglycolic acid was determined to be $10.6,{ }^{12}$ whereas the $\mathrm{pK}_{\mathrm{a}}$ for the thiol group of covalently attached TGA was calculated to be 8.52 utilizing the ACD software program (Toronto, Canada) Acciordingly, 100-fold more thiolate anions, $-S^{-}$, representing the reactive form of thiol moieties for an oxidation process, are available on the conjugate compared to unconjugated TGA leading to a rapid oxidation at $\mathrm{pH} 6$. The results of this study therefore provide strong evidence for the formation of amide bonds between chitosan and TGA during the coupling reaction, as this 
significant change in the reactivity of the thiol groups of TGA can only be explained by an alteration in its chemical structure

Figure 3. Comparison of the disulfide bond formation kinetic within $0.5 \%$ $(\mathrm{m} / \mathrm{v})$ chitosan-TGA conjugate $(\mathrm{O})$ and a $0.5 \%(\mathrm{~m} / \mathrm{v})$ chitosan / TGA mixture (匹). Studies were performed at $\mathrm{pH} 60$ and $37^{\circ} \mathrm{C}$. Indicated values are means $( \pm \mathrm{SD})$ of at least three experiments

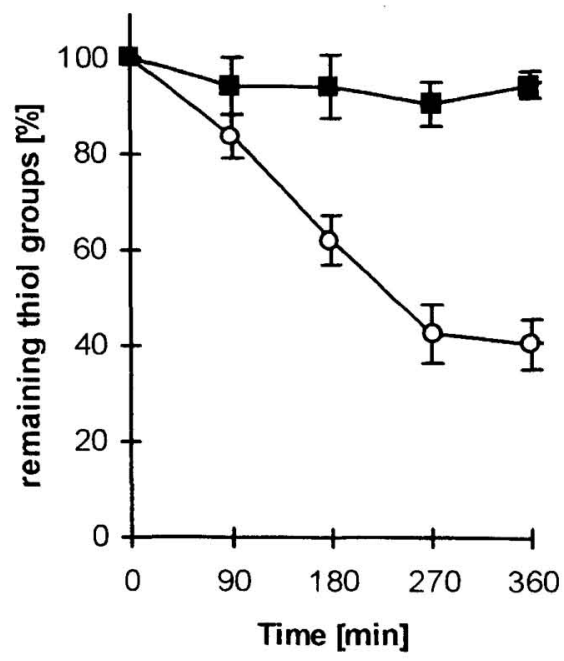

\section{Cysteine and cysteine methyl ester binding studies}

The capability of chitosan-TGA to bind cysteine and cysteine methyl ester was investigated by simple diffusion experiments carried out at $\mathrm{pH} \mathrm{5.5.} \mathrm{Results} \mathrm{of} \mathrm{this} \mathrm{study} \mathrm{demonstrated,} \mathrm{that} \mathrm{the}$ binding of cysteine to thiolated chitosan is a strongly time dependent process. Within four hours half of the cysteine was bound to the polymer conjugate. The thiolate anions of cysteine on the one hand and of the chitosan-TGA conjugate on the other hand are thereby essential for the formation of disulfide bonds. According to the $\mathrm{pK}_{\mathrm{a}}$ of the thiol group of cysteine and the chitosan-TGA conjugate determined to be 8.35 and $8.5,{ }^{13}$ using the equation: $f_{\text {ion }}=100 /\left[1+10^{(\mathbf{p H}+\mathbf{p K a})}\right],{ }^{14}$ only $0.14 \%$ and $0.10 \%$ of all thiol groups are available as anions at $\mathrm{pH} 5.5$, respectively Non covalent binding of cysteine to chitosan by simple ionic interactions between the carboxylic acid group of the sulfhydryl compound and the primary amino group of the polymer should be eliminated by high concentrations of acetic acid ( $1 \mathrm{M}$ ) during the diffusion studies. However, the results as shown in Fig. 4 demonstrated high ionic interactions between the sulfhydryl compounds and the polymers, as the amount of cysteine in the acceptor chamber was significantly lower when unmodified chitosan was added to the buffer solution in the donor chamber.

In contrast to cysteine, the cysteine methyl ester does not display a carboxylic acid group being responsible for ionic interactions with the primary amino groups of chitosan. Because of the exclusion of ionic interactions, relatively more of the sulfhydryl compound diffused into the 
acceptor chamber. Merely the addition of thiolated chitosan led to a pronounced retardation of cysteine methyl ester in the donor compartment. The results of this study are shown in Fig. 5.

Figure 4. Diffusion studies of L-cysteine. The donor chamber contained $0.04 \%(\mathrm{~m} / \mathrm{v})$ L-cysteine in combination with either $0.5 \%$ $(\mathrm{m} / \mathrm{v})$ chitosan-thioglycolic acid conjugate displaying $885 \mu \mathrm{mol}$ thiol groups / $\mathrm{g}$ polymer $(\mathbf{x}), \quad 0.5 \% \quad(\mathrm{~m} / \mathrm{v})$ chitosan hydrochloride $(\mathbf{\square}), 0.5 \% \quad(\mathrm{~m} / \mathrm{v})$ control polymer $(\Delta)$, or no polymer $(\bullet)$ in $1.0 \mathrm{M}$ acetate buffer $\mathrm{pH} 5.5$. Indicated values are means ( $\pm \mathrm{SD} ; \mathrm{n}=3-4$ ) of the L-cysteine concentration in the acceptor chamber expressed in percent, where 100\% represents the equilibrium concentration.
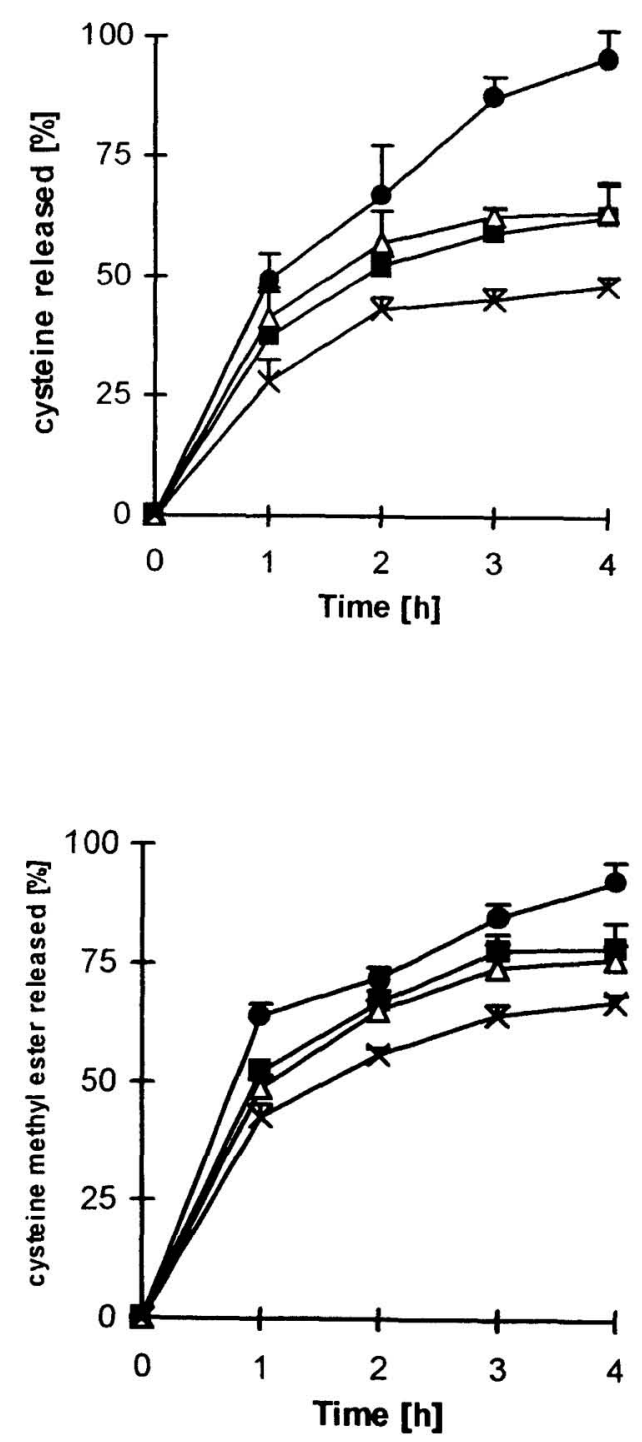

Figure 5. Diffusion studies of cysteine methyl ester. The donor chamber contained $0.04 \%(\mathrm{~m} / \mathrm{v})$ cysteine methyl ester in combination with either $0.5 \%(\mathrm{~m} / \mathrm{v})$ chitosanthioglycolic acid conjugate displaying $\mathbf{8 8 5}$ $\mu \mathrm{mol}$ thiol groups / $\mathrm{g}$ polymer (x), $0.5 \%$

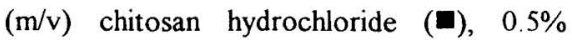
$(\mathrm{m} / \mathrm{v})$ control polymer $(\Delta)$, or no polymer $(\bullet)$ in $1.0 \mathrm{M}$ acetate buffer $\mathrm{pH} 5.5$. Indicated values are means $( \pm S D ; n=3-4)$ of the $L$ cysteine concentration in the acceptor chamber expressed in percent, where $100 \%$ represents the equilibrium concentration 


\section{Discussion}

The synthesis of chitosan-TGA conjugates via a carbodiimide offers the advantage that it can be performed in aqueous solutions without any toxic organic solvents which are difficult to completely remove. Furthermore, remaining traces of the carbodiimide are rapidly transformed into harmless urea derivatives. So far, however, the method seemed to be of minor practical relevance, as the yield of covalently attached thioglycolic acid was to low Although the synthesis has already been improved by optimizing the $\mathrm{pH}$ and the share of chitosan to TGA during the reaction, only $0.8 \%$ of the theoretical maximum of TGA was immobilized to the polymer. ${ }^{9}$ Raising the concentration of the carbodiimide, however, led to an almost exponential increase in covalently attached TGA rendering this synthesis useful for the production of chitosan-TGA conjugates in lager quantities.

Because of the covalent attachment of TGA to the polymer, the reactivity of its thiol groups being essential for the formation of covalent bonds to mucin glycoproteins and the formation of disulfide bonds within the polymeric network itself, was strongly improved. In addition the reactivity of the thiol groups was raised by increasing the $\mathrm{pH}$. Previous studies demonstrated the oxidation of $30 \%$ and $75 \%$ of thiol groups of a chitosan-TGA conjugate at $\mathrm{pH} 5.0$ and 6.5 within $6 \mathrm{~h}$, respectively. ${ }^{9}$ The decrease in thiol groups within the chitosan-TGA conjugate as shown in Fig 3 is thereby in good agreement with these results Moreover, the $\mathrm{pH}$-dependence of the reactivity of thiol groups was also demonstrated for anionic thiolated polymers such as polycarbophil-cysteine ${ }^{1}$ and carboxymethylcellulose-cysteine ${ }^{5}$

Apart from chemical modifications (I) and the pH of the medium (II), charge of neighboring groups to the sulfhydryl moiety (III) also has an impact on thiol reactivity. Thiol groups with an unionized or same charged local environment form disulfide bonds less rapidly than when surrounded by oppositely charged groups. ${ }^{15}$ For instance, Snyder et al. demonstrated that the negative sulfhydryl compound 5,5'-di-thiobis(2-nitrobenzoic acid) reacts most readily with cysteine derivatives whose immediate neighbors are positive and least readily with cysteine derivatives such as $\mathrm{N}$ acetylcysteine having a negative neighbor ${ }^{16}$ Accordingly, chitosan-TGA should form disulfide bonds less rapidly with itself or cysteine methyl ester than with cysteine exhibiting an anionic substructure (-COO') close to the thiol moiety. The results of diffusion studies with cysteine and cysteine methyl ester demonstrating a comparatively lower binding of the latter one are therefore in accordance with this theory. However, the lower binding of cysteine methyl ester can also be explained by the higher $\mathrm{pK}_{\mathrm{a}}$ of the thiol group of cysteine methyl ester. The $\mathrm{pK}_{\mathrm{a}}$ of the thiol group of cysteine is 8.35 , whereas it is 8.99 for its methyl ester ${ }^{17}$ Accordingly, cysteine displays more reactive thiolate anions being essential for the binding to chitosan-TGA at pH 5.5 than the cysteine methyl ester.

Besides diffusion studies as shown in Fig. 4 and 5 , the interaction between polymer immobilized thiol groups and cysteine moieties of mucus glycoproteins was demonstrated for polycarbophil- 
cysteine conjugates. Aqueous homogenates of polycarbophil and porcine mucus were separated from each other by centrifugation, whereas thiolated polycarbophil could not be separated any more from the mucus. Moreover, the mucoadhesive properties of thiolated polycarbophil were very strongly reduced in the presence of the reducing agent dithiothreitol, excluding the formation of disulfide bonds.

Because of its availability via an efficient synthetic pathway and its high reactive thiol groups being responsible for improved mucoadhesive properties and cohesive properties, chitosan-TGA seems to be a promising new excipient for various drug delivery systems.

\section{Experimental}

\section{Synthesis of chitosan- thioglycolic acid conjugates}

In order to obtain a $1 \%(\mathrm{~m} / \mathrm{v})$ solution of chitosan hydrochloride $0.5 \mathrm{~g}$ of chitosan (chitosan from crab shell; degree of deacetylation $>85 \%$; SIGMA, St. Louis, MO) were hydrated in $4 \mathrm{ml}$ of $1 \mathrm{M}$ $\mathrm{HCl}$ and dissolved in $46 \mathrm{ml}$ of demineralized water. Insoluble impurities were removed by filtration. The polymer solution was dialyzed against demineralized water for 24 hours in order to remove also polymer fragments of low molecular weight and to adjust the $\mathrm{pH}$ of chitosan hydrochloride to 5. Afterwards, $0.5 \mathrm{~g}$ of sodium thioglycolate (NaTGA; molecular mass: 114.1; SIGMA, St. Louis, MO) were dissolved in the dialysate under stirring and 1-ethyl-3-(3dimethylaminopropyl)carbodiimide hydrochloride (EDAC; Sigma, St. Louis, MO) dissolved in 1 $\mathrm{ml}$ of demineralized water was added in a final concentration of $50,75,100$, or $125 \mathrm{mM}$. The $\mathrm{pH}$ was adjusted to 5.0 with $1 \mathrm{M} \mathrm{HCl}$ and the reaction mixtures were incubated for three hours at room temperature under vigorous stirring. Samples prepared in exactly the same way as the polymerTGA conjugates but omitting EDAC during the coupling reaction served as controls for the following analytical studies. Unbound TGA and the coupling reagent were eliminated by dialyzing the reaction mixtures at $10^{\circ} \mathrm{C}$ in the dark first against $5 \mathrm{mM} \mathrm{HCl}$, two times against the same medium but containing $1 \% \mathrm{NaCl}$, and then exhaustively two times against $5 \mathrm{mM} \mathrm{HCl}$. Samples and controls were frozen at $-20^{\circ} \mathrm{C}$ until further use

\section{Determination of the final polymer concentration}

In order to quantify the polymer concentration in the final dialysate, $10 \mathrm{ml}$ aliquots were lyophilized by drying frozen aqueous polymer solutions at $-30^{\circ} \mathrm{C}$ and 0.01 mbar (Christ Beta 1-8K; Germany) and determining the amount of remaining polymer gravimetrically 


\section{Determination of the thiol group content}

The degree of modification was determined by quantifying the amount of thiol groups on the chitosan-TGA conjugates and controls. To $200 \mu \mathrm{l}$ samples of the conjugates and the control solutions, $200 \mu \mathrm{l}$ of $0.5 \mathrm{M}$ phosphate buffer and $200 \mu \mathrm{l}$ of Ellman's reagent [3 $\mathrm{mg}$ of 5,5'dithiobis(2-nitrobenzoic acid) (Sigma, St. Louis, MO) dissolved in $10 \mathrm{ml}$ of $0.5 \mathrm{M}$ phosphate buffer $\mathrm{pH}$ 8.0] were added The reaction was allowed to proceed for $90 \mathrm{~min}$ at room temperature Afterwards, the precipitated polymer was removed by centrifugation $(24,000 \mathrm{~g} ; 5 \mathrm{~min})$ and $300 \mu \mathrm{l}$ of the supernatant fluid was transferred to a microtitration plate and the absorbance was immediately measured at $450 \mathrm{~nm}$. The amount of thiol moieties was calculated from an according standard curve obtained by chitosan solutions with increasing amounts of TGA

\section{Comparison of the disulfide bond formation kinetic within chitosan-TGA conjugate and a chitosan / TGA mixture}

A solution of the chitosan-thioglycolic acid conjugate displaying the highest amount of covalently attached thiol groups and a $1 \%(\mathrm{~m} / \mathrm{v})$ chitosan hydrochloride solution were both diluted with demineralized water and $0.5 \mathrm{M}$ phosphate buffer $\mathrm{pH} 6.0$ in order to obtain $0.5 \%(\mathrm{~m} / \mathrm{v})$ polymer solutions in $50 \mathrm{mM}$ phosphate buffer $\mathrm{pH} 6.0$. To the $0.5 \%$ chitosan hydrochloride solution TGA was added in a concentration corresponding to the amount immobilized on the conjugate. Both samples were incubated at $37^{\circ} \mathrm{C}$ under shaking. At predetermined time points, aliquots of $200 \mu \mathrm{l}$ were withdrawn, $100 \mu \mathrm{l}$ of $0.5 \mathrm{M}$ phosphate buffer $\mathrm{pH} 6.0$ and $200 \mu \mathrm{l}$ of Ellman's reagent were added, and the amount of remaining thiol groups was determined as described above

\section{Diffusion studies}

To $0.5 \%(\mathrm{~m} / \mathrm{v})$ chitosan-thioglycolic acid conjugate displaying the highest amount of covalently attached thiol groups, $0.5 \%(\mathrm{~m} / \mathrm{v})$ chitosan hydrochloride, and $0.5 \%(\mathrm{~m} / \mathrm{v})$ of the control polymer or

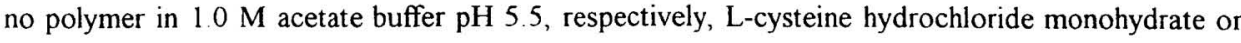
L-cysteine methyl ester was added in a final concentration of $0.04 \%(\mathrm{~m} / \mathrm{v})$. The reaction mixtures were preincubated for one hour at room temperature and then transferred to the donor chamber of an Ussing-type diffusion cell. The acceptor chamber was filled with one $\mathrm{ml}$ of $1.0 \mathrm{M}$ acetate buffer pH 5.5. Dialysis tubings (molecular mass cut-off: $10 \mathrm{kDa}$ ) served as semipermeable membrane. At predetermined time points aliquots of $200 \mu \mathrm{l}$ were withdrawn from the acceptor chamber and substituted by $1.0 \mathrm{M}$ acetate buffer $\mathrm{pH} 5.5$

The amount of diffused cysteine or cysteine methyl ester was determined with 2,4,6trinitrobenzenesulfonic acid (TNBS). To $100 \mu \mathrm{l}$ of withdrawn samples $100 \mu \mathrm{l}$ of $8 \% \mathrm{NaHCO}_{3}$ and $100 \mu \mathrm{l}$ of $0.2 \%$ TNBS (Sigma, St. Louis, MO) were added. Reaction mixtures were incubated at $37^{\circ} \mathrm{C}$ for 2 hours followed by measuring the absorbance at $450 \mathrm{~nm}$ (Lambda 16; Perkin-Elmer, Vienna, Austria). The amount of cysteine or cysteine methyl ester developing with the TNBS- 
reagent an orange dye was calculated utilizing standard curves of increasing amounts of cysteine or cysteine methyl ester.

\section{Statistical data analysis}

Statistical data analysis was performed using the Student $t$ test with $p<0.05$ as the minimal level of significance

Acknowledgements: This work was supported by Grant No. P13820-MOB from the Fonds zur Förderung der wissenschaftlichen Forschung (FWF) to A. Bernkop-Schnürch.

\section{References}

1. Bernkop-Schnürch A., Schwarz V., Steininger S. (1999), Pharm. Res. 16: 876

2 Bernkop-Schnürch A., Steininger S. (2000), Int. J. Pharm. 194: 239

3. Bernkop-Schnurch A., in Dumitriu, S. (Ed.): Polymeric Biomaterials, 2. Ed., Marcel Dekker, New York 2000, in press

4. Gum J.R., Hicks Jr. J.W., Toribara N.W., Rothe E.-M., Lagace R.E., Kim Y.S. (1992), J. Biol Chem. 267: 21375

5. Bernkop-Schnurch A., Scholler S., Biebel R.G. (2000), J. Control Release 66: 39

6. Bernkop-Schnurch A., Thaler S. (2000), J. Pharm. Sci. 89: 901

7. Clausen A.E., Bernkop-Schnürch A. (2000), J. Pharm. Sci. 89: 1253

8 Marschütz M.K., Caliceti P., Bernkop-Schnürch A. (2000), Pharm. Res. 17: 1468

9. Kast C.E., Bernkop-Schnürch A: Biomaterials, in press.

10. Holmberg K, Hansen B. (1979), Acta Chem. Scand. B 33: 410

11. Staros J.V., Wright R.W., Swingle D.M. (1986), Analytical Biochemistry 156: 220

12. Wrathall D.P., Izatt R.M., Christensen J.J. (1964), J. Amer. Chem. Soc. 86: 4779

13. Roth H.J., Eger K., Troschutz, R.: Pharmazeutische Chemie II: Arzneistoffanalyse, (3. Ed.) p. 188 Thieme Verlag, Stuttgart, Germany 1990

14. Martin A., Swarbrick J., Cammarata A.: Physikalische Pharmazie, p. 225, Wissenschaftliche Verlagsgesellschaft, Stuttgart, Germany 1975

15. Snyder G.H., Cennerazzo M.J., Karalis A.J., Field D. (1981), Biochemistry 20: 6509

16. Snyder G.H., Reddy M.K., Cennerazzo M.J., Field D. (1983), Biochim Biophys Acta 749 219

17. Li N.C., Manning R.A. (1955), J. Am. Chem. Soc. 77: 5225 